\title{
Morphometric changes correlate with poor psychological outcomes in patients with acromegaly
}

\author{
Syed Ali Imran, Jitske Tiemensma', Stephanie M Kaiser, Michael Vallis, \\ Steve Doucette, Ebad Abidi, Churn-Ern Yip, Barna De Tugwell, Ferhan Siddiqi \\ and David B Clarke ${ }^{\dagger}$ \\ Dalhousie University, 6299 South Street, Halifax, Nova Scotia, Canada B3H 4R2 and 'SSHA, Psychological Science, \\ University of California, Merced, 5200 North Lake Road, Merced, California 95343, USA \\ ${ }^{\dagger} \mathrm{D}$ B Clarke is now at Division of Neurosurgery, Dalhousie University and Queen Elizabeth II Health Sciences Centre, \\ 1796 Summer Street, Suite 3807, Halifax, Nova Scotia, Canada B3H 3A7
}

Correspondence should be addressed to D B Clarke Email d.clarke@dal.ca

\begin{abstract}
Objective: Acromegaly is frequently associated with altered facial appearance at the time of diagnosis. Furthermore, acromegaly is also associated with adverse psychological outcomes. We conducted a single-centre, cross-sectional study comparing patients with growth hormone vs non-functioning pituitary adenomas (NFA) to assess the association between morphometric changes and psychological outcomes and illness perception of patients with acromegaly.

Methods: A seven-step scale was developed to grade morphometric changes based on facial photographs. In addition, all patients were asked to draw an image of their own body and an image of what they considered to be an average healthy body and complete seven psychological questionnaires. We recruited 55 consecutive patients in each of the two groups who had undergone surgery with or without radiation therapy (RT).

Results: Our data showed that the clinician-rated morphometric scale was highly reliable in assessing facial changes, with 93/99 (Intraclass correlation coefficient $(I C C)=0.95(0.93-0.97)$ ) graded as similar by independent raters. The mean (s.D.) grading for Acro and NFA patients on the clinician-rated morphometric scale were $3.5(1.3)$ and $0.41(0.35)$ respectively $(P<0.0001)$. A higher clinician-rated morphometric score was also predictive of a poorer score on the drawing test.

Conclusions: Our study demonstrates a correlation between physical changes associated with acromegaly and poor psychological outcomes, whereas no such correlation existed with modes of therapy, disease control status, RT, malignancy, initial or recent GH/IGF1 or secondary hormonal deficiency. Our data support the utility of the morphometric scale as a clinical tool for grading facial changes.
\end{abstract}

\section{Introduction}

Acromegaly is associated with typical morphometric changes, including altered facial appearance and enlarged extremities. These changes are generally insidious in nature, which on average can delay the diagnosis for about 12 years (1). Consequently, $98 \%$ of patients with acromegaly have significant morphometric changes at the time of diagnosis (2). An association between acromegaly and adverse psychological outcomes has been known for many decades
(C) 2016 European Society of Endocrinology Printed in Great Britain
(3). Several studies have demonstrated an increased prevalence of psychopathology, cognitive dysfunction and personality changes both in patients with active acromegaly $(4,5,6,7)$ as well as those in remission (8). In addition, patients with acromegaly have a negative self-perception based on the drawing test (9), a novel technique that can illustrate their notions in a more concrete and specific way than questionnaires or structured interviews limited by

Published by Bioscientifica Ltd. 
operating along predefined dimensions $(10,11)$. The drawings can also be useful in identifying idiosyncratic beliefs, which can play a critical role in illness perceptions, medication adherence, self-management skills and quality of life (QoL) (12). To date, no study has looked at the association between morphometric changes and psychological outcomes in acromegaly. We intended to examine the pathological personality traits, psychological experiences (mood, QoL, self-perception and social support) and biomedical markers in association with morphometric changes and the drawing test. We conducted a study with a threefold objective: i) to develop a clinician rating scale to quantify morphometric changes in acromegaly; ii) to compare the clinician-rated morphometric scale with patients' illness perception based on a drawing test and iii) to determine the association between morphometric changes and psychological outcomes.

\section{Subjects and methods}

\section{Patients}

This was a single-centre, cross-sectional cohort study conducted at the Halifax Neuropituitary Program, Dalhousie University, Halifax, Nova Scotia, Canada. We recruited 55 consecutive patients in each of the two groups: i) patients with acromegaly (Acro group) who had undergone surgery with or without radiation therapy (RT) and/or medical therapy and ii) patients with nonfunctioning pituitary adenomas (NFA) group who had undergone surgery with or without RT. None of the patients approached for the study declined to participate.

The following data were extracted for all patients: current age, gender, age at diagnosis, time since diagnosis, date of surgery, date of RT (if given), complete pituitary hormonal profile at presentation and within 3 months of the study visit, history of malignancy, secondary hormonal insufficiency and replacement therapy for secondary hormonal insufficiency. For the Acro group, the following additional variables were collected: fasting growth hormone (GH), serum insulin-like growth factor 1 (IGF1) and post-75 g oral glucose tolerance test (OGTT) GH at diagnosis and within 3 months of the study visit and the date of start and type of medical therapy.

The diagnosis of acromegaly had been established on the basis of typical clinical features, an elevated IGF1 and non-suppressible GH to $<1 \mu \mathrm{g} / \mathrm{l}$ after $75 \mathrm{~g}$ OGTT. Patients with normal serum IGF1 and GH $<1 \mu \mathrm{g} / \mathrm{l}$ after OGTT and not requiring medical therapy were regarded as in remission; those who achieved normal serum IGF1 but required medical therapy were regarded as controlled and those who had an elevated IGF1 despite maximum medical therapy were regarded as uncontrolled. Adrenal insuffciency was defined as either basal serum cortisol of $<130 \mathrm{nmol} / 1$ or failure of serum cortisol to rise $>500 \mathrm{nmol} / \mathrm{l}$ after an insulin tolerance test or $250 \mu \mathrm{g}$ ACTH stimulation test; secondary hypothyroidism diagnosis was based on a free thyroxine below the normal reference range with normal or low thyroid-stimulating hormone. Secondary hypogonadism in men was defined as total serum testosterone below the normal reference range with normal or below-normal serum luteinizing hormone (LH) and follicle-stimulating hormone (FSH), whereas in premenopausal women it was defined as amenorrhea for $>6$ months (after excluding pregnancy and birth control therapy), below-normal serum estradiol and inappropriate normal or low serum LH and FSH, whereas in postmenopausal women it was defined as below-normal serum LH and FSH in the absence of estrogen therapy. Diabetes insipidus was diagnosed based on the presence of polyuria and polydipsia in addition to abnormal water deprivation test. We do not routinely perform dynamic testing for $\mathrm{GH}$ deficiency unless coverage for GH therapy is available; therefore, GH deficiency was not consistently evaluated.

\section{Photographs}

Each patient had a lateral and a three-quarter frontal view (Fig. 1) photograph taken. All photographs were taken by a Canon EOS Rebel t5i - 18 MP dSLR with dedicated flash and diffuser. Capture mode was set to RAW/Jpeg quality 8 and ISO was 200/DIN 24. The white balance was synched
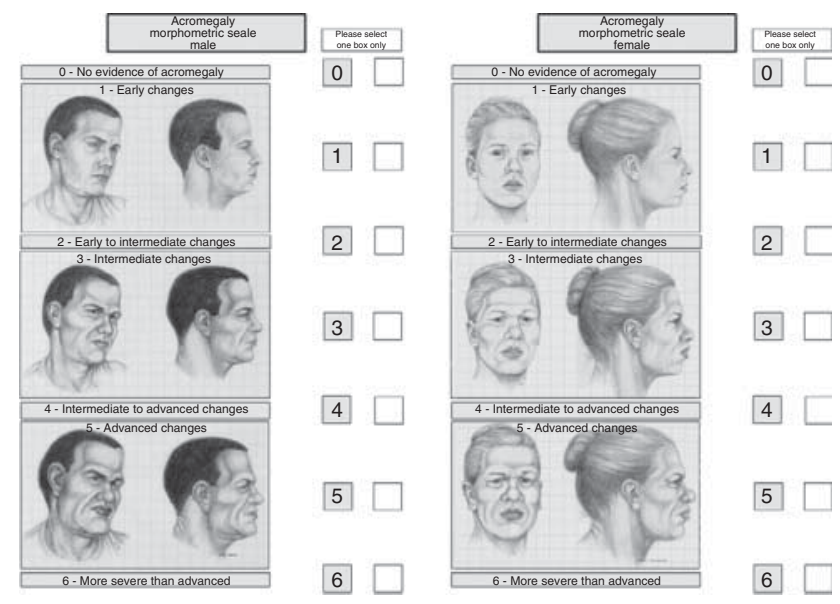
0 $1 \square$ 2 3. 4 . 5 6

Figure 1

Clinician-rated morphometric scale. 
with the dedicated flash at $\sim 6000 \mathrm{~K}$. The subjects were seated $24^{\prime \prime}$ in front of a white screen and the camera was placed $48^{\prime \prime}$ away from the subjects. Focal length was set at $80 \mathrm{~mm}$, aperture setting was f8 and shutter speed was $1 / 125 \mathrm{~s}$. Dedicated flash was set to f11 at $1 / 125 \mathrm{~s}$ with diffuser in place. All photos were printed on semi-gloss photo quality paper at $9 \times 3 \mathrm{~cm}$.

\section{Clinician-rated morphometric scale}

In order to develop a clinician-rated visual morphometric scale, we requested current and all previous photographs from each patient's personal collection from the time of graduation from high school for patients with acromegaly, as well as 20 (ten of each gender) NFA patients, and received an average of seven photographs spanning a mean period of 40.2 years. These photographs were analyzed by a forensic artist to develop a seven-step scale (Fig. 1). The scale contained four primary categories: no evidence of acromegaly (0), early changes of acromegaly (1), intermediate changes of acromegaly (3) and advanced changes of acromegaly (5). Furthermore, due to subtle individual variations in morphometric features, there were three additional categories for in-between patients: early to intermediate changes (2), intermediate to advanced changes (4) and more severe than advanced changes (6). Two physicians (S A Imran and D B Clarke), who had been following all patients, independently graded each photograph based on the scale. Grading was regarded as similar if both physicians were within one step of each other. Where grading was more than one step apart, a consensus grading score was determined. This score was regarded as the expert grading score. Subsequently, four endocrinologists (S M Kaiser, F Siddiqi, B De Tugwell and C-E Yip) who were completely unfamiliar with the patients independently graded each photograph. Grading within one step of each other was regarded as similar and in case of a difference greater than one step, the panel met to reach a consensus grading. This was regarded as the non-expert grading score. Finally, the non-expert grading scores were compared against the expert scores and in case of greater than one step difference, the final consensus score was sought through discussion. If consensus could not be reached, the grading was regarded as divergent.

\section{Drawing test}

All patients were asked to make two drawings (Fig. 2) in a box of $20 \times 20 \mathrm{~cm}$ with the following instructions:

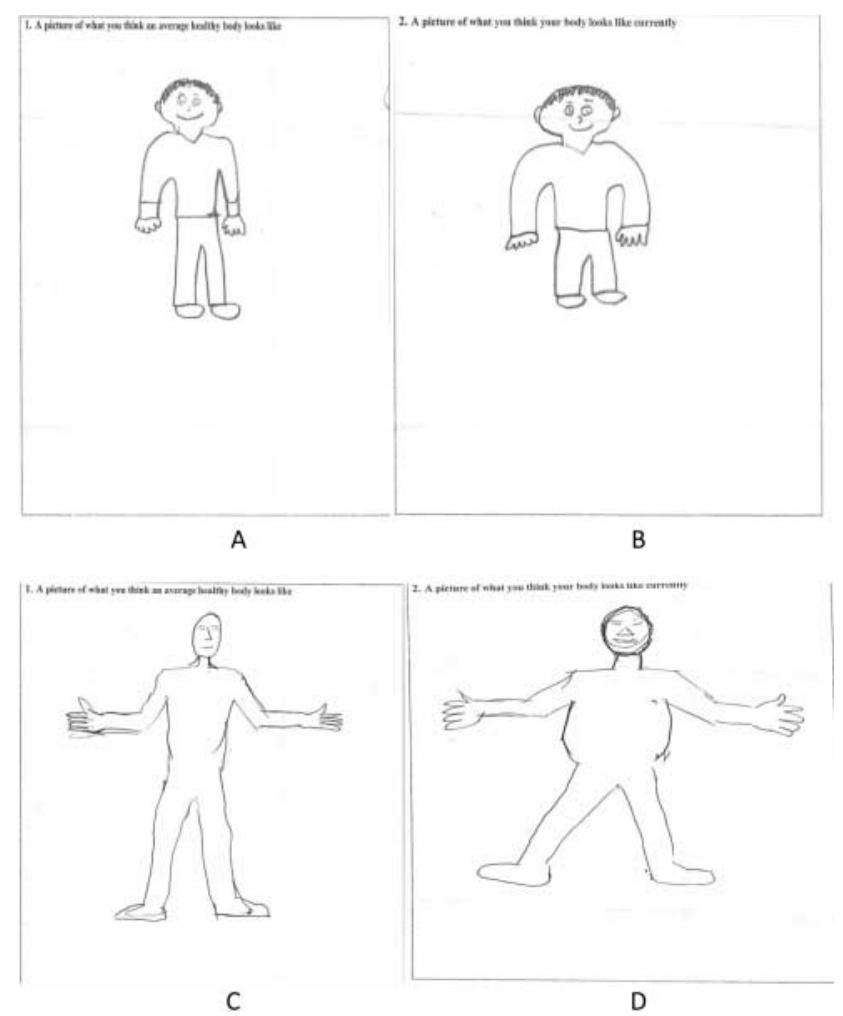

Figure 2

Self-drawing samples of NFA ( $A$ and $B$ ) and acromegaly (C and D).

drawing 1 - 'picture of what you think your body looks like currently' and drawing 2 - 'a picture of what you think an average healthy body looks like'. The drawings were scanned and imported into the freely available NIH ImageJ Software. The outside parameters of the drawing, head, right hand, and right foot were computed based on previously described methodology (9) by measuring in pixels and two independent observers scored the characteristics of the drawings. Subsequently, $\kappa$ scores were calculated for each characteristic. If the $\kappa$ was $<0.7$, consensus was reached between the raters by re-analyzing the drawings together. In addition, delta values were calculated based on percentage increase or decrease with the formula ((drawing $2 /$ drawing 1$)-1) \times 100)$ to score the difference between drawing 1 (own body) and drawing 2 (healthy body).

\section{Psychological questionnaires}

All patients were asked to complete seven questionnaires listed below. Psychological measures were divided into two types; personality pathology (used in most of the previously published literature) and current psychological 
experience, which address emotional functioning, QoL, self-perceptions and social support.

Personality pathology Dimensional Assessment of Personality Pathology-Short Form (DAPP-SF) (13). This is a 136 item self-report scale that assesses 18 dimensions of personality functioning that has been used in the acromegaly population by Tiemensma et al. (8). The score for each subscale differs with maxima of 30-40, and higher scores indicate more pronounced maladaptive personality traits. There are no formal cut-off scores for these subscales.

\section{Psychological experience}

Emotional functioning - Apathy scale $>$ This is a 14-item scale (items scored from 0 to 4 ) that has been validated in this population (14). The scale measures different features of apathy in the two previous weeks. The total score of this scale ranges from 0 to 42 points, with higher scores indicating greater apathy. Apathy is defined by a total score $\geq 14$ points.

Irritability scale - This is a 14-item scale similar in style to the Apathy scale (scored 0-4) that has been validated and used with acromegaly patients (15). The scale assesses different features of irritability in the two previous weeks. The total score ranges from 0 to 42 points, with higher scores indicating greater irritability. Irritability is defined by a total score $\geq 14$ points.

Quality of life $>$ Acromegaly QoL scale $>$ The AcroQoL consists of 22 questions on a five-point Likert scale. The response choices are divided into frequency of occurrence (ranging from 'always' to 'never') and degree of agreement with the items (ranging from 'completely agree' to 'completely disagree'). The total score ranges from 0 to 100 , with a lower score indicating a greater impact on health related QoL. The scale has been validated in a Spanish population and translated into English (16).

Self-perception $、$ The Rosenberg self-esteem scale $\rightarrow$ This is one of the well-used scales to assess overall self-esteem. It is a ten-item scale, with each item scored on a four-point scale (17). The total score is used to index self-esteem, with no cut-offs or population norms.

Body image distortion - This is a 14-item satisfaction with appearance scale, which assesses subjective satisfaction and social-behavioural impact; it has been validated (18) and the total score is used with no cut-off or population norms.

Social support $\rightarrow$ The Interpersonal Support Evaluation List The Interpersonal Support Evaluation List (ISEL) is a well established measure of social support that has been validated in the literature is a 40 -item self report scale, with four subscales: appraisal, tangible, self-esteem and belonging (19). The total scores are used with no clinical cut-offs or population norms.

\section{Statistical analysis}

Patient characteristics at presentation were presented as means and s.D.s for continuous variables and as frequencies with percentages for categorical data. Comparisons were assessed using the Student's $t$-test and the Fisher's exact test. The agreement between expert and non-expert scores were assessed using the intraclass correlation coefficient (20). Expert and non-expert clinician-rated morphometric scale scores were calculated as the mean of both physicians, and four independent endocrinologists respectively. The proportion of divergent scores between experts and non-experts were described with divergent scores represented as any rating difference $>1$ on the morphometric scale.

Psychological outcomes in NFA and Acro patients were expressed as means and s.D.s. The relationship between the clinician-rated morphometric scale and the psychological outcome scores were first described using receiver operating characteristic (ROC) curves and then expressed as means and s.D.s categorized into those scoring $<2$ vs $>2$, values that were determined based on

Table 1 Patient characteristics.

\begin{tabular}{|c|c|c|c|}
\hline Characteristics & $\begin{array}{c}\text { NFA } \\
(n=48)\end{array}$ & $\begin{array}{c}\text { Acro } \\
(n=51)\end{array}$ & $P$ value \\
\hline Female $(\%)$ & $25(52.1 \%)$ & $28(54.9 \%)$ & 0.84 \\
\hline Current age & $60.1 \pm 12.3$ & $58.3 \pm 14.6$ & 0.51 \\
\hline Age at presentation & $53.0 \pm 14.7$ & $46.0 \pm 14.0$ & 0.04 \\
\hline Years since diagnosis & $7.1 \pm 5.8$ & $11.4 \pm 7.3$ & 0.002 \\
\hline Stereotactic radiation therapy & $9(18.8 \%)$ & $19(40.4 \%)$ & 0.021 \\
\hline \multicolumn{4}{|c|}{ IGF1 levels } \\
\hline$\%$ ULN at presentation & NA & $171.6 \pm 112.2$ & \\
\hline$\%$ ULN at most recent visit & NA & $-10.9 \pm 32.7$ & \\
\hline IGF1 controlled/remission & & $42(89.4 \%)$ & \\
\hline IGF1 uncontrolled & & $5(10.6 \%)$ & \\
\hline Hypoadrenalism & $16(33.3 \%)$ & $8(15.7 \%)$ & 0.06 \\
\hline Hypothyroidism & $32(66.7 \%)$ & $24(47.1 \%)$ & 0.07 \\
\hline Hypogonadism & $24(50.0 \%)$ & $22(43.1 \%)$ & 0.13 \\
\hline Diabetes & $4(8.3 \%)$ & $2(3.9 \%)$ & 0.43 \\
\hline Malignancy & $4(8.3 \%)$ & $6(11.8 \%)$ & 0.74 \\
\hline
\end{tabular}


Table 2 Psychological outcomes in patients with acromegaly vs NFA. Data are presented as mean \pm s.D.

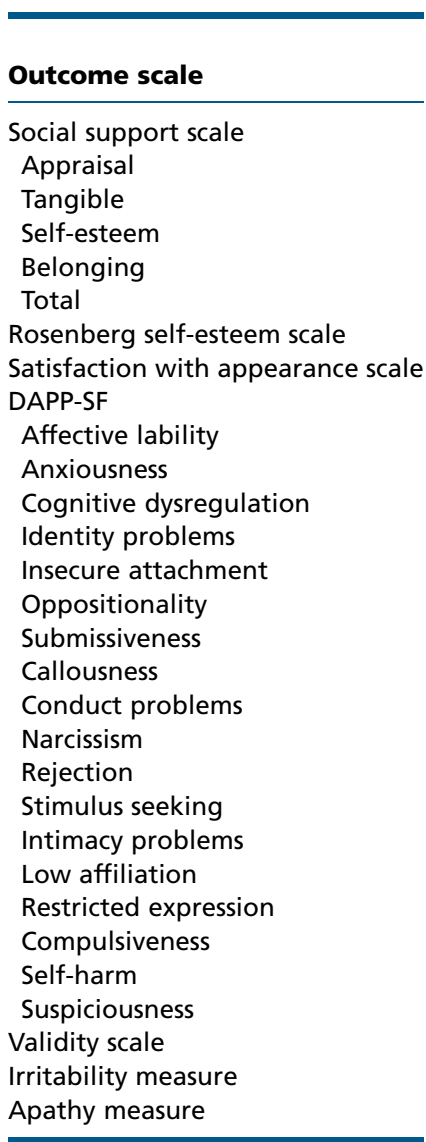

(OR) and 95\% CI. All analyses were completed using SAS version 9.4 (Cary, NC, USA).

\section{Results}

\section{Patient characteristics}

These are summarized in Table 1 . A total of 110 patients (55 in each group) were consecutively recruited for the study. Of those, 51 in the Acro group and 48 in the NFA group completed the study. The mean ages and gender distribution of the two groups were similar. The mean age at presentation in the NFA group was 53.0 (14.7) years compared with 46.0 (14.0) years in the Acro group $(P=0.04)$. Of all Acro patients, 42 were either controlled or in remission while nine were uncontrolled. There was no significant difference overall in the risk of secondary hormonal deficiency or malignancy between the two groups. All patients with secondary hormonal deficiency were on stable replacement therapy for at least 6 months and none of those patients were receiving active medical therapy for cancer and all malignancies were in remission for at least 24 months.

\section{Reliability of the morphometric scale}

Overall, there was robust agreement amongst physicians' grading based on the clinician-rated morphometric scale.
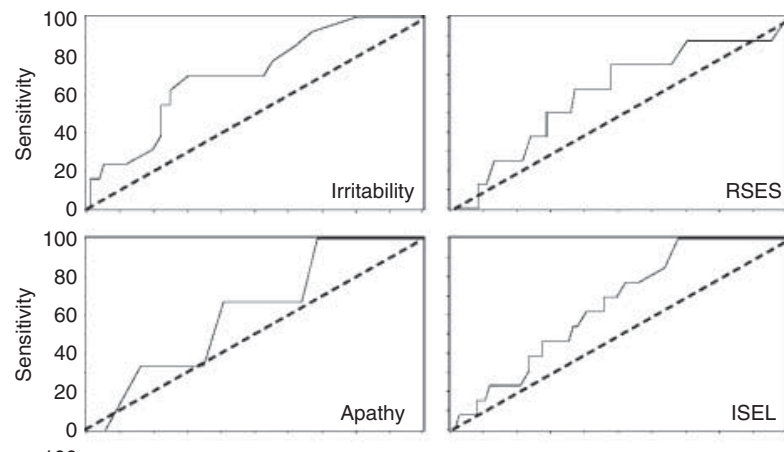

maximizing both sensitivity and specificity simultaneously from all ROC curves. A comparison in psychological outcomes was made for those with large delta values on the patient drawing test and categorized into those who had delta scores $>25 \%$ on any of the five categories (height, width, head width, right hand and right foot) vs those who did not, using the Student's t-test.

Correlations between the clinician-rated morphometric scores and the five individual delta values on the drawing test were summarized using Pearson's $R$. We also assessed the association between clinician-rated morphometric scores using logistic regression analysis and an ROC curve with the dependant categorized as those who had delta scores $>25 \%$ on any of the five categories as previously defined. Results are presented as odds ratio

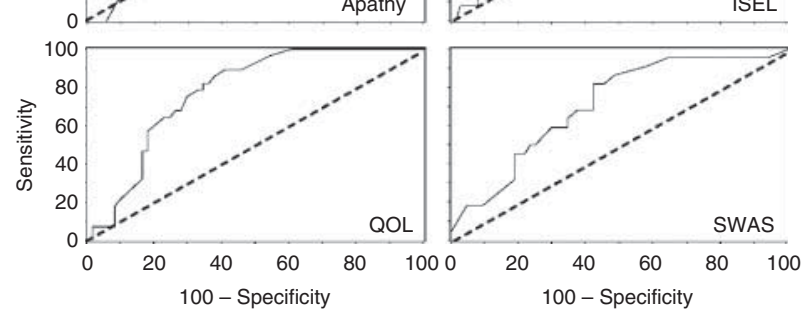

Figure 3

ROC curves: mean physician score as a predictor of poor psychological outcome. 
Before consensus, 73/99 (ICC $=0.83(0.72-0.89)$ ) were regarded as similar, whereas after consensus 93/99 $(\mathrm{ICC}=0.95(0.93-0.97))$ were similar with 6/99 being divergent. When comparing post-consensus grades between experts and non-experts, 20 (20.2\%) were identical, $73(73.7 \%)$ were within one step of each other and $6(6.1 \%)$ were within two steps of each other. None of the grades were more than two steps apart.

\section{Impact of acromegaly on psychological outcomes}

There data are summarized in Table 2. Acro patients were more likely to display affective lability, cognitive dysregulation, identity problems, intimacy problems and low affiliation than NFA patients (all $P<0.05$ ). Similarly, Acro patients scored higher on the measure of emotional apathy $(P=0.007)$, had lower QoL as measured by the total QoL scale $(P<0.0001)$, as well as the physical, appearance and personal relationships subscales (all $P<0.01)$, compared with NFA patients. Finally, acromegaly patients were less satisfied with their appearance $(P<0.0001)$ and had lower self-esteem $(P=0.005)$ than NFA patients.

Women, compared with men, reported lower QoL on the appearance scale (61.5 vs $74.3, P=0.007)$ as well as lower intimacy scores on the DAPP-SF $(P=0.005)$, whereas men were more likely to have conduct problem $(P=0.006)$ and callousness $(P=0.001)$ on the DAPP-SF. There were no significant relationships between poor psychological outcomes and the risk of malignancy, modes of therapy including surgery, medical therapy and RT, acromegaly control state (remission, controlled or uncontrolled), serum IGF1 levels either recent of at presentation or secondary hormonal deficiency status (data not shown).

Table 3 Adverse psychological outcomes in patients scoring 2 or higher on clinician-rated morphometric scale. Data are presented as mean \pm s.D.

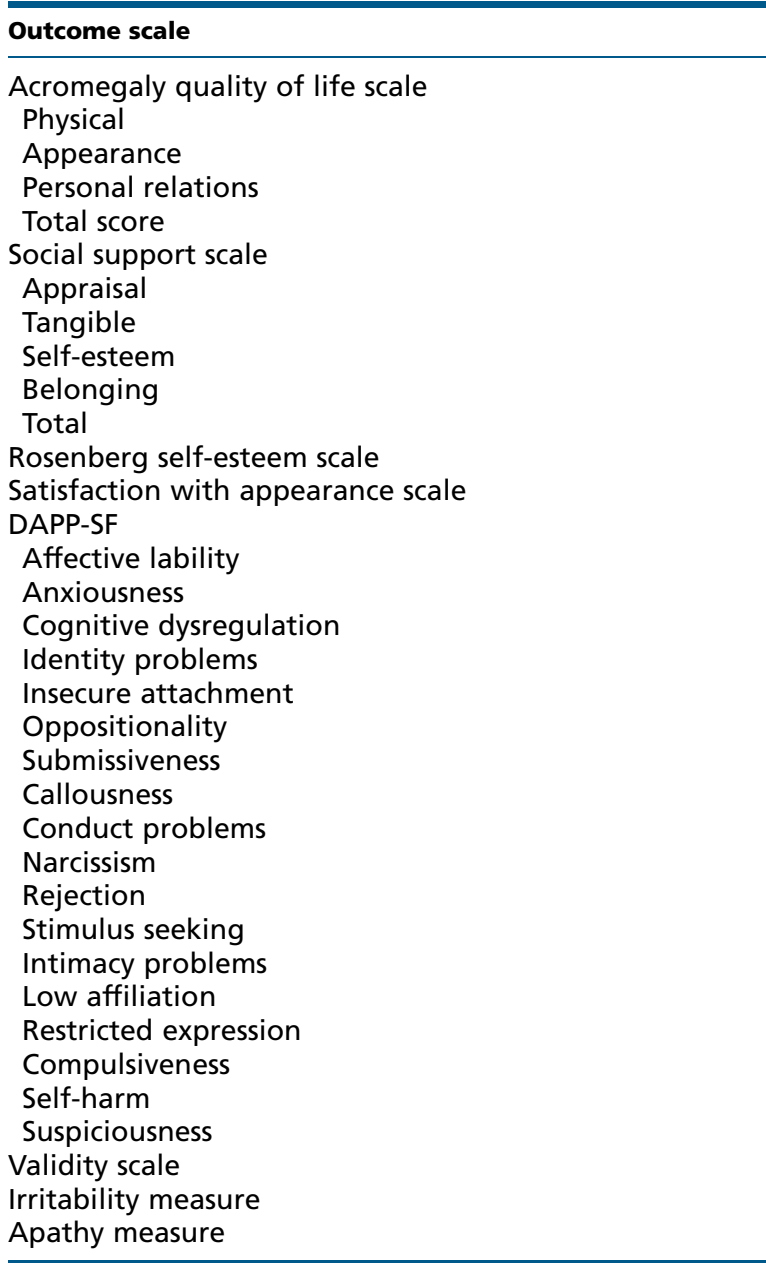

${ }^{*} P<0.05,{ }^{\dagger} P<0.01$ and ${ }^{\ddagger} P<0.0001$

\begin{tabular}{c}
\hline Score $>\mathbf{2}(n=45)$ \\
\\
$54.7 \pm 23.1$ \\
$56.5 \pm 21.2$ \\
$72.8 \pm 22.5$ \\
$61 \pm 20$ \\
$23.5 \pm 4.7$ \\
$25.5 \pm 3.3$ \\
$20.5 \pm 4.4$ \\
$23.4 \pm 4.4$ \\
$23.2 \pm 3.5$ \\
$21.5 \pm 5.8$ \\
$25.7 \pm 16.6$ \\
\\
$41.7 \pm 12.3$ \\
$39.8 \pm 13$ \\
$33.9 \pm 9.9$ \\
$35.5 \pm 12.2$ \\
$37 \pm 10.7$ \\
$37.1 \pm 10.4$ \\
$38.1 \pm 10$ \\
$28 \pm 7.5$ \\
$25.8 \pm 8$ \\
$34.1 \pm 8$ \\
$37.2 \pm 9.9$ \\
$35.5 \pm 7.4$ \\
$37.7 \pm 10.3$ \\
$38.6 \pm 11.3$ \\
$44 \pm 9.2$ \\
$52.3 \pm 11.3$ \\
$15.7 \pm 6.7$ \\
$29.5 \pm 8.4$ \\
$28.3 \pm 3.9$ \\
$11.9 \pm 8.3$ \\
$13.6 \pm 6.6$ \\
\\
\\
\\
\end{tabular}

\begin{tabular}{c}
\hline Score $<2(n=54)$ \\
\hline \\
$76.1 \pm 21.4$ \\
$77.6 \pm 19.3$ \\
$86.6 \pm 17.6$ \\
$79.9 \pm 17.4$ \\
$25.6 \pm 4.6$ \\
$26.6 \pm 4.2$ \\
$21.6 \pm 3.9$ \\
$24.9 \pm 4.6$ \\
$24.7 \pm 3.6$ \\
$24.1 \pm 4.7$ \\
$15.1 \pm 16.5$ \\
$38.3 \pm 10.3$ \\
$38.5 \pm 11.3$ \\
$29.3 \pm 7.1$ \\
$31.1 \pm 9.1$ \\
$34.5 \pm 9.9$ \\
$36.1 \pm 10$ \\
$36.3 \pm 8.1$ \\
$27.8 \pm 6.8$ \\
$23.9 \pm 6.3$ \\
$37.1 \pm 8.9$ \\
$38.7 \pm 9.2$ \\
$36.7 \pm 8.1$ \\
$32 \pm 9.2$ \\
$34.1 \pm 9.7$ \\
$39.9 \pm 8.6$ \\
$52.3 \pm 8.9$ \\
$13.7 \pm 3.8$ \\
$27.4 \pm 7.3$ \\
$28.3 \pm 4$ \\
$9.9 \pm 6.4$ \\
$10.2 \pm 4.9$ \\
\\
\\
\\
\\
\end{tabular}

\begin{tabular}{l}
\hline P value \\
\hline \\
$<0.0001^{\ddagger}$ \\
$<0.0001^{\ddagger}$ \\
$0.002^{\dagger}$ \\
$<0.0001^{\ddagger}$ \\
\\
$0.03^{*}$ \\
0.16 \\
0.21 \\
0.12 \\
0.06 \\
$0.02^{*}$ \\
$0.004^{\dagger}$ \\
\\
0.16 \\
0.61 \\
$0.01 *$ \\
0.05 \\
0.26 \\
0.62 \\
0.36 \\
0.91 \\
0.2 \\
0.09 \\
0.48 \\
0.45 \\
$0.007^{\dagger}$ \\
$0.04^{*}$ \\
$0.03^{*}$ \\
0.98 \\
0.09 \\
0.22 \\
0.93 \\
0.21 \\
$0.006^{\dagger}$ \\
\end{tabular}




\section{Psychological outcomes and the clinician-rated morphometric scale}

The mean (s.D.) grading for Acro and NFA patients on the clinician-rated morphometric scale were 3.5 (1.3) and 0.41 $(0.35)$ respectively $(P<0.0001)$. Individuals with a higher morphometric score were more likely to score high on apathy, less satisfaction with personal appearance, poorer personal relationships, poorer QoL as measured by the total QoL scale, as well as the physical, appearance and personal relationships subscales (all $P<0.01$ ), lower levels of self-esteem $(P=0.02)$ and reduced appraised social support $(P=0.03)$.

An attempt was made to determine a cut-off score on the clinician-rated morphometric scale that might index psychological disturbance. A score of $>2$ on the clinicianrated morphometric scale was associated with an increased likelihood of poor outcomes (Fig. 3 and Table 3). The sensitivity values for various outcomes in individuals who received a grading score $>2$ were: irritability (69.2\%), apathy $(66.7 \%)$, Acro QOL total score $(78.6 \%)$, social support (62.5\%), Rosenberg self-esteem scale (61.5\%) and satisfaction with appearance (68.2\%). When looking at the Acro group alone, data were divided on the basis of median morphometric reading of 3.5. There was a trend towards positive correlation between higher morphometric reading and adverse psychological outcomes, especially in conduct problem $(P<0.05)$ and suspiciousness $(P=0.05)$.

\section{Psychological outcomes and the drawing test}

Compared with NFA patients, Acro patients had a significantly higher distortion in the right hand (47.52

Table 4 Adverse psychological outcomes in patients $<25 \%$ distortion vs $>25 \%$ distortion in the drawing test. Data are presented as mean \pm s.D.

\begin{tabular}{l}
\hline \\
Outcome scale \\
\hline Acromegaly quality of life scale \\
Physical \\
Appearance \\
Personal relations \\
Total score \\
Social support scale \\
Appraisal \\
Tangible \\
Self-esteem \\
Belonging \\
Total \\
Rosenberg self-esteem scale \\
Satisfaction with appearance scale \\
DAPP-SF \\
Affective lability \\
Anxiousness \\
Cognitive dysregulation \\
Identity problems \\
Insecure attachment \\
Oppositionality \\
Submissiveness \\
Callousness \\
Conduct problems \\
Narcissism \\
Rejection \\
Stimulus seeking \\
Intimacy problems \\
Low affiliation \\
Restricted expression \\
Compulsiveness \\
Self-harm \\
Suspiciousness \\
Validity scale \\
Irritability measure \\
Apathy measure \\
\hline
\end{tabular}

\begin{tabular}{c}
\hline One or more delta value \\
$>\mathbf{2 5 \%}(n=44)$ \\
\\
\\
$58.4 \pm 24.1$ \\
$59.8 \pm 19.9$ \\
$75.3 \pm 22.5$ \\
$64.2 \pm 19.8$ \\
$23.6 \pm 5$ \\
$25.5 \pm 4$ \\
$20 \pm 4.2$ \\
$23 \pm 5.3$ \\
$23 \pm 4$ \\
$20.8 \pm 5.7$ \\
$22.9 \pm 17.3$ \\
$42 \pm 11.7$ \\
$42 \pm 11.3$ \\
$32.6 \pm 9.1$ \\
$36.3 \pm 12$ \\
$36.7 \pm 10.9$ \\
$37.9 \pm 9.1$ \\
$39.9 \pm 9.3$ \\
$27.5 \pm 7.3$ \\
$25.1 \pm 7$ \\
$35.8 \pm 7.9$ \\
$37.1 \pm 9.2$ \\
$36.1 \pm 7.1$ \\
$36.3 \pm 10.7$ \\
$38.8 \pm 11$ \\
$42.4 \pm 9.6$ \\
$52.3 \pm 10.6$ \\
$16.2 \pm 6.9$ \\
$28.3 \pm 8.2$ \\
$28.5 \pm 4.1$ \\
$12.6 \pm 7.6$ \\
$12.7 \pm 5.8$ \\
\end{tabular}

All delta values
$<\mathbf{2 5 \%}(n=26)$

$81 \pm 17.8$
$83.9 \pm 14.7$
$90.6 \pm 12.5$
$85 \pm 13.5$
$25.1 \pm 4.9$
$26.4 \pm 3.6$
$22 \pm 3.2$
$25.4 \pm 3.5$
$24.7 \pm 3.1$
$25.8 \pm 3.1$
$15.2 \pm 16.4$
$36.4 \pm 9.9$
$35.6 \pm 12.6$
$28.2 \pm 8.3$
$29.3 \pm 6.3$
$32.8 \pm 8.3$
$34.8 \pm 10.5$
$33.8 \pm 6.2$
$28.5 \pm 6.5$
$24.3 \pm 7.1$
$34 \pm 6.8$
$37.6 \pm 10.5$
$36 \pm 8.7$
$33.3 \pm 6.5$
$32.7 \pm 6.9$
$40.4 \pm 7.9$
$51.5 \pm 9.8$
$12.8 \pm 1.5$
$28.1 \pm 8.6$
$28.5 \pm 3.5$
$8.3 \pm 5.8$
$9.3 \pm 4.1$

\begin{tabular}{|c|}
\hline$P$ value \\
\hline $\begin{array}{r}0.0001^{\dagger} \\
<0.0001^{\ddagger} \\
0.0006^{\dagger} \\
<0.0001^{\ddagger}\end{array}$ \\
\hline $\begin{array}{c}0.23 \\
0.35 \\
0.04^{*} \\
0.02^{*} \\
0.07 \\
<0.0001^{\ddagger} \\
0.09\end{array}$ \\
\hline $\begin{array}{l}0.045^{\star} \\
0.03^{\star} \\
0.046^{\star} \\
0.002^{\dagger} \\
0.12 \\
0.2 \\
0.002^{\dagger} \\
0.58 \\
0.65 \\
0.35 \\
0.83 \\
0.96 \\
0.15 \\
0.006^{\dagger} \\
0.38 \\
0.75 \\
0.003^{\dagger} \\
0.9 \\
0.97 \\
0.02^{\star} \\
0.01^{\star}\end{array}$ \\
\hline
\end{tabular}

${ }^{*} P<0.05,{ }^{\dagger} P<0.01$ and ${ }^{\ddagger} P<0.0001$ 
(57.94) vs $-2.70(28.82) ; P=0.005)$, right foot $(34.11$ (57.29) vs 8.10 (34.34); $P=0.002)$ and height (6.89 (22.41) vs $-1.62(19.90) ; P=0.017)$, whereas there was no significant difference of distortion in Acro vs NFA groups in width (16.58 (38.39) vs 5.40 (37.41)) and head width $(18.72$ (41.56) vs 9.94 (28.87)). When comparing individuals who had $>25 \%$ distortion in any single category (height, width, head width, right hand and right foot) vs those who had $<25 \%$ distortion in all categories, there was a significantly higher risk of adverse outcomes on various scales (see Table 4).

\section{Correlation between the drawings and the clinician-rated morphometric scale}

Overall, there was a positive correlation between the drawing test and the clinician-rated morphometric scale. The correlations between the clinician-rated morphometric scale and the drawing delta value were: height, $r=0.23$ ( $P=0.06)$; width, $r=0.19(P=0.12)$; head width, $r=0.06(P=0.61)$; right foot, $r=0.25(0.08)$ and right hand, $r=0.33(P=0.02)$. A higher clinician-rated morphometric score was predictive of a higher delta score on the drawing test with an increase in the odds (OR 1.47, 95\% CI 1.08$2.00, P=0.02)$ of $>25 \%$ delta scores on one of the patient drawing scores (Fig. 4).

\section{Discussion}

Acromegaly is associated with progressive morphometric changes such as acral enlargement, fleshy lips,

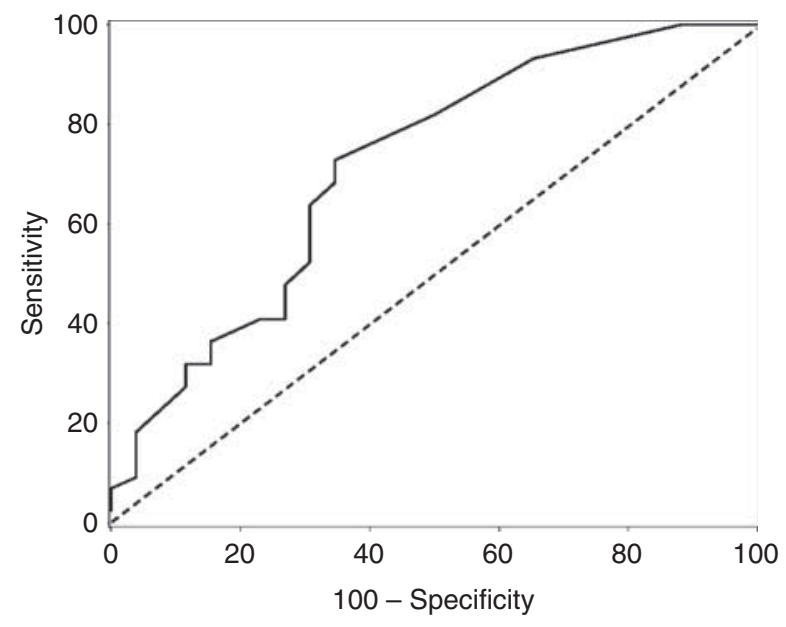

Figure 4

ROC curve: mean physician score as a predictor of one or more drawing delta $>25 \%$. enlargement of the nose, prominence of the skull and jawbones as well as skin and soft tissue changes that can progress in time to significant disfigurement. Furthermore, many of these changes are only partially reversible and most patients, even after achieving biochemical control, never achieve their pre-disease features. The emotional and psychological effects of such lingering changes have so far not been thoroughly studied.

Previous studies have reported an increased risk of psychopathology and maladaptive personality traits in patients with active acromegaly and those who have been in long-term remission $(8,21,22)$. However, to date there has been no satisfactory explanation of this phenomenon except that it may be related to irreversible changes associated with previous GH excess (8). We extended this research by examining not only pathological personality traits but psychological experiences (mood, QoL, selfperception and social support) as well as biomedical markers (length of disease, GH and IGF1 levels on initial presentation and at the last visit, disease control status, modes of therapy including surgery, medical and RT, malignancy as well as secondary hormonal deficiency). None of the biomedical markers were associated with personality pathology or psychological experience although others have reported that younger age, RT and higher BMI are associated with poor QoL in Acro patients $(23,24)$. Larger studies utilizing more sensitive questionnaires are required to assess subtle effects of disease on psychological wellbeing of these patients. For instance, a recent study (25) suggested that the three subscale model of the Acro QoL reflecting physical complaints, appearance issues and personal relations issues was better than the traditional total score in reflecting multidimensional measures of QoL. In our study, acromegaly negatively impacted a number of aspects of psychological functioning related to emotions (apathy), QoL, satisfaction with appearance and self-esteem.

Interestingly, there was a distinct gender association with certain psychological outcomes. Our data showed that women, compared with men, had lower appearancerelated QoL, whereas men were more likely to have conduct features and callousness. These differences, if replicated, might suggest that the ongoing negative sequelae of acromegaly impact men and women differently. Women might internalize the distress of the condition and, hence, their lower satisfaction with appearance. Men, on the other hand, might externalize this stress, acting out and becoming less caring. Small sample size precluded us from assessing the effects of estrogen or testosterone replacement on various 
psychological outcomes. However, larger studies may provide a better insight into these trends.

One of the important outcomes of this study was the development of a clinician-rated scale to assess the extent of morphometric changes in acromegaly. This scale demonstrated good reliability between raters and correlated with adverse psychological outcomes. Similarly, selfdrawings of discrepancy between perceived and ideal body image also correlated with psychological distress. These data, if replicated in larger series, will lend credibility to the interpretation that the psychological disturbance associated with acromegaly may be mediated by morphometric changes that are experienced by the individual as distressing.

Although our data showed a correlation between the physician grading and patient self-drawing, it appears that the morphometric scale and the drawing tests seem to be associated with slightly different outcomes. This is an interesting finding that needs to be replicated. The selfdrawings were related to personality pathology more so than the clinician ratings. Both were predictive of psychological distress. Further research is needed to examine whether the different measures of morphometric distortion predict different outcomes and/or respond differently to interventions.

Our morphometric scale showed a high reliability, in that $94 \%$ of the photographs were graded within one step, whereas none was graded with a discrepancy of more than two steps. Furthermore, when comparing Acro with NFA patients, our scale suggested a distinct cut-off that predicted high risk of adverse psychological outcomes. A grade $>2$ was highly predictive of an increased risk of adverse outcomes. If confirmed in larger future studies, these data indicate potential value in the clinical application of this morphometric scale as a sensitive and reliable tool to identify high-risk patients. The selfdrawing, on the other hand, requires interpretation of the test by a special computer program that may limit its utility in a busy clinical setting.

While we are unable to conclude that there is a causal relationship between morphometric changes and adverse psychological outcomes due to the design of our study, it certainly is a tantalizing hypothesis. An irreversible effect of excess $\mathrm{GH}$ on cognitive functioning cannot be excluded (26), but future studies should also focus on various interventions, both psychological and cosmetic, to assess the impact of such strategies on improving psychopathological outcomes.

Our study has several strengths. We had a closely matched group of control patients and, unlike a previous study where only $52 \%$ of the invited patients participated (9), none of the invited patients declined the study and furthermore, $87 \%$ patients in the NFA group and $92 \%$ in the Acro group completed the study. However, there were limitations as well. This was a single-centre study with a relatively small population of patients. Furthermore, two physicians (S A Imran and D B Clarke) were very familiar with patients, which may have impacted their assessment of patient photographs; to counterbalance this, the remaining physicians were truly blinded to the underlying diagnosis.

In summary, our study demonstrates a correlation between physical changes associated with acromegaly and poor psychological outcomes, whereas no such association existed with other disease-related variables. Future studies are needed to assess the validity of the morphometric scale in a larger and diverse population of patients and to assess the effect of intervention on outcomes identified through this scale.

Declaration of interest

The authors declare that there is no conflict of interest that could be perceived as prejudicing the impartiality of the research reported.

\section{Funding}

This research was funded by the Capital Health Research Fund

\section{References}

1 Colao A, Ferone D, Marzullo P \& Lombardi G. Systemic complications of acromegaly: epidemiology, pathogenesis, and management. Endocrine Reviews 200425 102-152. (doi:10.1210/er.2002-0022)

2 Molitch ME. Clinical manifestations of acromegaly. Endocrinology and Metabolism Clinics of North America 199221 597-614.

3 Bleuler M. Personality changes in pituitary disorders. BMJ 19511 580-581. (doi:10.1136/bmj.1.4706.580)

4 Richert S, Strauss A, Lierheimer A, Eversmann T \& Fahlbusch R. Psychopathology, mental functions and personality in patients with acromegaly. Acta Endocrinologica. Supplementum 198325333.

5 Sablowski N, Pawlik K, Ludecke DK \& Herrmann HD. Aspects of personality in patients with pituitary adenomas. Acta Neurochirurgica 198683 8-11. (doi:10.1007/BF01420501)

6 Flitsch J, Spitzner S \& Lüdecke DK. Emotional disorders in patients with different types of pituitary adenomas and factors affecting the diagnostic process. Experimental and Clinical Endocrinology \& Diabetes 2000108 480-485. (doi:10.1055/s-2000-8144)

7 Tanriverdi F, Yapislar H, Karaca Z, Unluhizarci K, Suer C \& Kelestimur F. Evaluation of cognitive performance by using P300 auditory event related potentials (ERPs) in patients with growth hormone $(\mathrm{GH})$ deficiency and acromegaly. Growth Hormone \& IGF Research 200919 24-30. (doi:10.1016/j.ghir.2008.05.002)

8 Tiemensma J, Biermasz NR, van der Mast RC, Wassenaar MJE, Huub A, Middelkoop H, Pereira A \& Romjin J. Increased psychopathology and maladaptive personality traits, but normal cognitive functioning, in patients after long-term cure of acromegaly. Journal of Clinical 
Endocrinology and Metabolism 201095 E392-E402. (doi:10.1210/ jc.2010-1253)

9 Tiemensma J, Pereira AM, Romijn JA, Broadbent E, Biermasz NR \& Kaptein AA. Persistent negative illness perceptions despite long-term biochemical control of acromegaly: novel application of the drawing test. European Journal of Endocrinology 2015172 583-593. (doi:10.1530/ EJE-14-0996)

10 Broadbent E, Ellis CJ, Gamble G \& Petrie KJ. Changes in patient drawings of the heart identify slow recovery after myocardial infarction. Psychosomatic Medicine 200668 910-913. (doi:10.1097/01. psy.0000242121.02571.10)

11 Reynolds L, Broadbent E, Ellis CJ, Gamble G \& Petrie KJ. Patients' drawings illustrate psychological and functional status in heart failure. Journal of Psychosomatic Research 200763 525-532. (doi:10.1016/ j.jpsychores.2007.03.007)

12 Broadbent E, Petrie KJ, Ellis CJ, Ying J \& Gamble G. A picture of health myocardial infarction patients' drawings of their hearts and subsequent disability: a longitudinal study. Journal of Psychosomatic Research 2004 57 583-587. (doi:10.1016/j.jpsychores.2004.03.014)

13 van Kampen D, de Beurs E \& Andrea H. A short form of the Dimensional Assessment of Personality Pathology-Basic Questionnaire (DAPP-BQ): the DAPP-SF. Psychiatry Research 2008160 115-128. (doi:10.1016/ j.psychres.2007.05.002)

14 Starkstein SE, Mayberg HS, Preziosi TJ, Andrezejewski P, Leiguarda R \& Robinson RG. Reliability, validity, and clinical correlates of apathy in Parkinson's disease. Journal of Neuropsychiatry and Clinical Neurosciences 19924 134-139. (doi:10.1176/jnp.4.2.134)

15 Chatterjee A, Anderson KE, Moskowitz CB, Hauser WK \& Marder KS. A comparison of self-report and caregiver assessment of depression, apathy, and irritability in Huntington's disease. Journal of Neuropsychiatry and Clinical Neurosciences 200517 378-383. (doi:10.1176/jnp.17.3.378)

16 Webb SM, Prieto L, Badia X, Catalá M, Gaztambide S, Lucas T, Páramo C, Picó A, Lucas A, Halperin I et al. Acromegaly Quality of Life Questionnaire (ACROQOL) a new health-related quality of life questionnaire for patients with acromegaly: development and psychometric properties. Clinical Endocrinology 200257 251-258. (doi:10.1046/j.1365-2265.2002.01597.x)

17 Crandal R. The measurement of self-esteem and related constructs. In Measures of Social Psychological Attitudes. Revised Edition, pp 80-82. Eds JP Robinson \& PR Shaver. Ann Arbor: ISR, 1973.
18 Lawrence WJ, Heinberg LJ \& Roca R. Development and validation of the satisfaction with appearance scale: assessing body image among burninjured patients. Psychological Assessment 1998 10 64-70. (doi:10.1037/ 1040-3590.10.1.64)

19 Cohen S \& Hoberman H. Positive events and social supports as buffers of life change stress. Journal of Applied Social Psychology 198313 99-125. (doi:10.1111/j.1559-1816.1983.tb02325.x)

20 Shrout PE \& Fleiss JL. Intraclass correlations: uses in assessing rater reliability. Psychological Bulletin 197986 420-428. (doi:10.1037/ 0033-2909.86.2.420)

21 Sonino N, Ruini C, Navarrini C, Ottolini F, Sirri L, Paoletta A, Fallo F, Boscaro M \& Fava GA. Psychosocial impairment in patients treated for pituitary disease: a controlled study. Clinical Endocrinology $2007 \mathbf{6 7}$ 719-726. (doi:10.1111/j.1365-2265.2007.02951.x)

22 Sievers C, Dimopoulou C, Pfister H, Lieb R, Steffin B, Roemmler J, Schopohl J, Mueller M, Schneider HJ, Ising M et al. Prevalence of DSMIV mental disorders in acromegaly: a cross-sectional study in 81 acromegalic patients. Clinical Endocrinology 200971 691-701. (doi:10.1111/j.1365-2265.2009.03555.x)

23 Yoshida K, Fukuoka H, Matsumoto R, Bando H, Suda K, Nishizawa $H$, Iguchi G, Ogawa W, Webb SM \& Takahashi Y. The quality of life in acromegalic patients with biochemical remission by surgery alone is superior to that in those with pharmaceutical therapy without radiotherapy, using the newly developed Japanese version of the AcroQoL. Pituitary 201518 876-883. (doi:10.1007/ s11102-015-0665-2)

24 Conaglen HM, de Jong D, Crawford V, Elston ME \& Conaglen JV. Body image disturbance in acromegaly patients compared to nonfunctioning pituitary adenoma patients and controls. International Journal of Endocrinology 20152015 624872. (doi:10.1155/2015/624872)

25 Felt JM Jr, Depaoli SA, Pereira AM, Biermasz NR \& Tiemensma J. Total score or subscales in scoring the acromegaly quality of life questionnaire: using novel confirmatory methods to compare scoring options. European Journal of Endocrinology 2015173 37-42. (doi:10.1530/EJE-15-0228)

26 Martin-Rodriguez JF, Madrazo-Atutxa A, Venegas-Moreno E, Benito-López P, Gálvez MÁ, Cano DA, Tinahones FJ, Torres-Vela E, Soto-Moreno A \& Leal-Cerro A. Neurocognitive function in acromegaly after surgical resection of GH-secreting adenoma versus naive acromegaly. PLoS ONE 20138 e60041. (doi:10.1371/journal. pone.0060041)

Received 3 September 2015

Revised version received 6 October 2015

Accepted 12 October 2015 